\title{
An infrared diagnostic for magnetism in hot stars ${ }^{\star}$ (Research Note)
}

\author{
M. E. Oksala ${ }^{1}$, J. H. Grunhut ${ }^{2}$, M. Kraus ${ }^{3,4}$, M. Borges Fernandes ${ }^{5}$, C. Neiner ${ }^{1}$, C. A. H. Condori ${ }^{5}$, \\ J. C. N. Campagnolo ${ }^{5}$, and T. B. Souza ${ }^{5}$
}

\author{
${ }^{1}$ LESIA, Observatoire de Paris, CNRS UMR 8109, UPMC, Université Paris Diderot, 5 place Jules Janssen, 92190 Meudon, France \\ e-mail: mary.oksala@obspm.fr \\ 2 European Southern Observatory, Karl-Schwarzschild-Str. 2, 85748 Garching, Germany \\ 3 Astronomický ústav, Akademie věd České republiky, Fričova 298, 25165 Ondřejov, Czech Republic \\ 4 Tartu Observatory, Tõravere, 61602 Tartumaa, Estonia \\ 5 Observatório Nacional, Rua General José Cristino, 77 São Cristovão, 20921-400 Rio de Janeiro, Brazil
}

Received 27 February 2015 / Accepted 28 April 2015

ABSTRACT

\begin{abstract}
Magnetospheric observational proxies are used for indirect detection of magnetic fields in hot stars in the X-ray, UV, optical, and radio wavelength ranges. To determine the viability of infrared (IR) hydrogen recombination lines as a magnetic diagnostic for these stars, we have obtained low-resolution $(R \sim 1200)$, near-IR spectra of the known magnetic B2V stars HR 5907 and HR 7355, taken with the Ohio State Infrared Imager/Spectrometer (OSIRIS) attached to the $4.1 \mathrm{~m}$ Southern Astrophysical Research (SOAR) Telescope. Both stars show definite variable emission features in IR hydrogen lines of the Brackett series, with similar properties as those found in optical spectra, including the derived location of the detected magnetospheric plasma. These features also have the added advantage of a lowered contribution of stellar flux at these wavelengths, making circumstellar material more easily detectable. IR diagnostics will be useful for the future study of magnetic hot stars, to detect and analyze lower-density environments, and to detect magnetic candidates in areas obscured from UV and optical observations, increasing the number of known magnetic stars to determine basic formation properties and investigate the origin of their magnetic fields.
\end{abstract}

Key words. stars: magnetic field - circumstellar matter - infrared: stars - stars: early-type - techniques: spectroscopic

\section{Introduction}

Extensive work over the past four decades has revealed the presence and general properties of large-scale magnetic fields in a subset of hot stars (see, e.g., Landstreet \& Borra 1978; Borra \& Landstreet 1979; Donati et al. 2002). Long-term studies demonstrate that these magnetic structures remain stable over long periods (see, e.g., Oksala et al. 2012; Silvester et al. 2014), making them ideal laboratories to examine the interaction of such fields with the moderate to strong stellar winds of OB stars. Competition between the strengths of the field and wind in the circumstellar environments of magnetic hot stars creates a magnetosphere, or a region above the stellar surface occupied by ionized gas (Babel \& Montmerle 1997; ud-Doula \& Owocki 2002). As the stellar wind drives material from the surface, the plasma follows the field lines from opposite footpoints to the top of the magnetic loop, where the two streams collide, cool, and then either are held up by centrifugal forces or fall back onto the surface of the star.

This material can be detected in a number of ways and thus can be used as a proxy for direct magnetic detection through spectropolarimetric observations (see, e.g., Petit et al. 2013, and references therein). In the optical, the cooler and denser postshock material trapped in the stellar magnetosphere is detected

$\star$ Based on observations obtained at the Southern Astrophysical Research (SOAR) telescope, which is a joint project of the Ministerio da Ciência, Tecnologia, e Inovação (MCTI) da República Federativa do Brasil, the US National Optical Astronomy Observatory (NOAO), the University of North Carolina at Chapel Hill (UNC), and Michigan State University (MSU). in the hydrogen recombination lines, typically $\mathrm{H} \alpha$ lines, although the emission pattern depends on the magnetic and stellar properties (i.e., field strength, mass-loss rate, stellar geometry; Oksala et al. 2012; Rivinius et al. 2013; Grunhut et al. 2012). Other physical manifestations of the magnetosphere can be detected in observations of X-ray (e.g., Gagné et al. 2005), UV (e.g., Henrichs et al. 1993), and radio (e.g., Trigilio et al. 2004), typically either presenting as peculiar features or variability. However, to date, there have been no dedicated studies of infrared (IR) spectral features to obtain analogous diagnostics for hot stars.

IR spectral studies of normal OB stars (e.g., Hanson et al. 2005; Najarro et al. 2011) show hydrogen recombination lines, either in emission or in absorption. Analyses of Be stars found emission combined with minimal photospheric contribution in IR hydrogen line profiles, allowing a more direct investigation of the circumstellar material than possible with optical features (see, e.g., Lenorzer et al. 2002; Granada et al. 2010). As magnetic OB stars also have circumstellar material and may show $\mathrm{H} \alpha$ emission (albeit typically weaker features), it would be logical to suggest that the IR spectra of magnetic stars similarly show strong emission features.

With the research above as motivation, we began a survey to investigate the IR spectral properties of known magnetic, hot stars. During the course of this work, Eikenberry et al. (2014) observed in their study of red giants as part of Sloan Digital Sky Survey III's Apache Point Observatory Galactic Evolution Experiment (SDSS-III/APOGEE, Eisenstein et al. 2011) two early B-type stars in the $H$-band as telluric standards, but found 
Table 1. Observation summary.

\begin{tabular}{lcc}
\hline \hline Object & HJD & Phase \\
\hline HR 5907 & 2456462.60178 & 0.42 \\
& 2456462.63069 & 0.48 \\
& 2456462.64967 & 0.51 \\
& 2456462.66552 & 0.55 \\
& 2456462.68113 & 0.58 \\
& 2456462.69877 & 0.61 \\
\hline HR 7355 & 2456462.72116 & 0.63 \\
& 2456462.73978 & 0.67 \\
& 2456462.75889 & 0.70 \\
& 2456462.78514 & 0.75 \\
\hline
\end{tabular}

that they contained strong hydrogen line emission with peak velocities far outside what would normally be expected for Be stars, leading the authors to conclude that these two stars were candidate magnetic B stars. The findings of Eikenberry et al. (2014) provided justification to continue development of our work.

Here, we present our pioneering pilot study into the viability of IR hydrogen recombination lines as a magnetic diagnostic for massive stars, examining time-series of two well-known magnetic chemically peculiar stars, HR 5907 and HR 7355.

\section{Targets and observations}

For this preliminary investigation, we chose two targets, the He-strong B2V stars HR 7355 and HR 5907, both known to possess strong magnetic fields and circumstellar material. A comprehensive study of HR 5907 by Grunhut et al. (2012) revealed a strong, primarily dipole magnetic field with a strength of $10.4 \mathrm{kG}$ and extremely rapid rotation $\left(v \sin i=290 \mathrm{~km} \mathrm{~s}^{-1}\right.$, $\left.P_{\text {rot }}=0.508276 \mathrm{~d}\right)$. The authors also found strong, variable $\mathrm{H} \alpha$ emission and a variable, optical photometric light curve, both indicating the presence of plasma trapped in a co-rotating (centrifugal) magnetosphere. Similarly, HR 7355 had been suggested to be a magnetic star by Rivinius et al. (2008), based on the appearance of $\mathrm{H} \alpha$ emission in its spectrum and a variable, optical photometric light curve. This notion was confirmed by Oksala et al. (2010), and Rivinius et al. (2010, 2013), which indicated that HR 7355 indeed possesses a primarily dipolar magnetic field with a strength of $11.6 \mathrm{kG}$. The star was also found to be rapidly rotating with $P_{\mathrm{rot}}=0.52144 \mathrm{~d}$ and $v \sin i=310 \mathrm{~km} \mathrm{~s}^{-1}$. Ultimately, the strength of the optical magnetospheric signatures of the two stars makes them ideal targets for identifying corresponding IR diagnostics.

To demonstrate the feasibility of such studies, we have obtained low-resolution $(R \sim 1200)$, near-IR spectra, taken with the Ohio State Infrared Imager/Spectrometer (OSIRIS) attached to the $4.1 \mathrm{~m}$ Southern Astrophysical Research (SOAR) Telescope. The data were obtained during the night of 2013 June 18 with a $1^{\prime \prime}$ slit width and in cross-dispersed mode, to simultaneously obtain $J_{-}, H$-, and $K$-band spectra. We obtained six sets of observations of HR 5907 and four for HR 7355, with each set consisting of four exposures in an ABBA nod pattern. Two additional spectra of HR 7355 were taken during worsening weather conditions and were in the end of too low quality to be considered. A summary of these observations is presented in Table 1. Rotational phases were computed using the ephemeris derived by Grunhut et al. (2012) for HR 5907 and that derived by Rivinius et al. (2013) for HR 7355. Individual exposure times for each ABBA position were $6 \mathrm{~s}$ for HR 5907 and $15 \mathrm{~s}$ for HR 7355.

The data were reduced using standard IRAF tasks within the echelle package, including flat-field division, sky-background
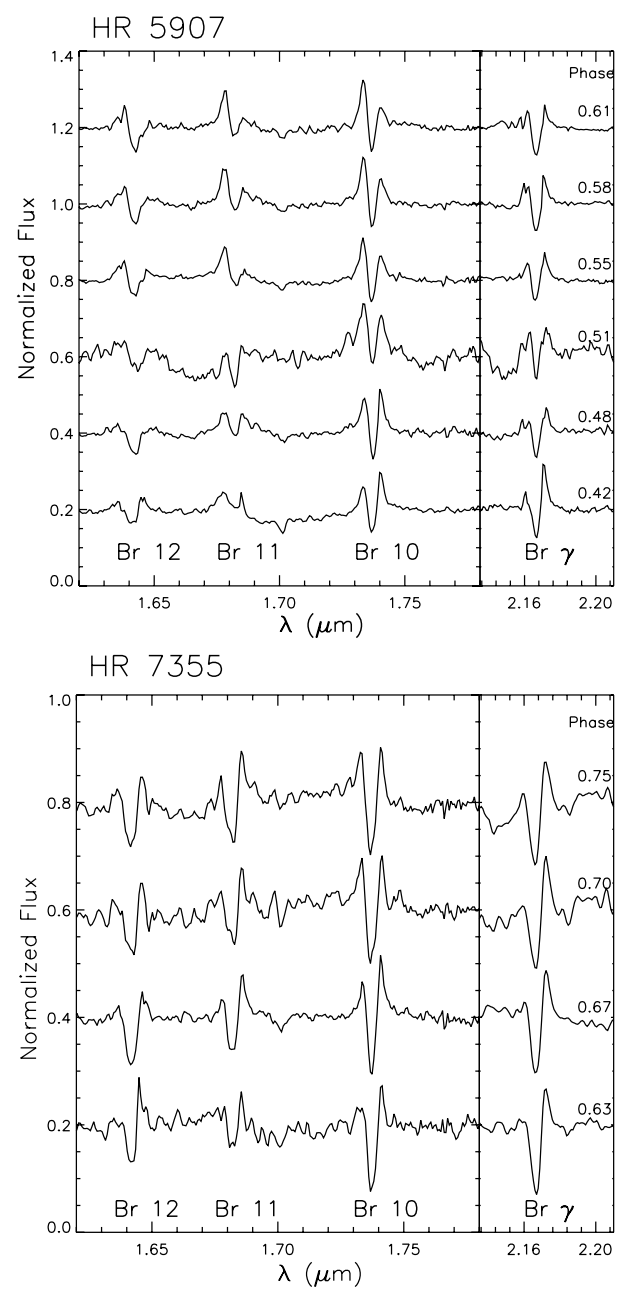

Fig. 1. Time-series of low-resolution $(R \sim 1200)$, near-IR spectra of the He-strong B2V stars HR 5907 (top) and HR 7355 (bottom). Stellar rotational phases for each spectrum are indicated on the right, and the hydrogen recombination lines are identified along the bottom.

subtraction, and wavelength calibration using ThAr spectra. A master flat image was obtained using the task xdflat within the program XDSpres, a CL script written for OSIRIS by Ruschel-Dutra et al. (2011). Spectra of the B9V stars HIP 78809 and HIP 93583 for HR 5097 and HR 7355, respectively, were obtained directly following the target at a similar airmass to minimize the effect of changing atmospheric conditions to create telluric templates. The IRAF task telluric used these templates to remove atmospheric lines from the target spectra. Spectra extracted from each set of ABBA exposures were co-added to increase the final signal-to-noise ratio $(\mathrm{S} / \mathrm{N})$, which ranges from $\sim 100$ to 200 .

Because of the wavelength range acquired and the location of a strong telluric region, the correction of atmospheric effects introduces uncertainties. The $J$-band spectra were unusable, and thus we proceeded using only the $H$ and $K$-bands. We note that due to the low resolution of the acquired data, we are only able to identify hydrogen recombination lines, and the $K$-band may still experience lingering effects, such that $\operatorname{Br} \gamma$ may yet be contaminated.

\section{Results and discussion}

Both HR 5907 and HR 7355 exhibit strong emission features in the line profiles of the IR hydrogen recombination lines. Figure 1 

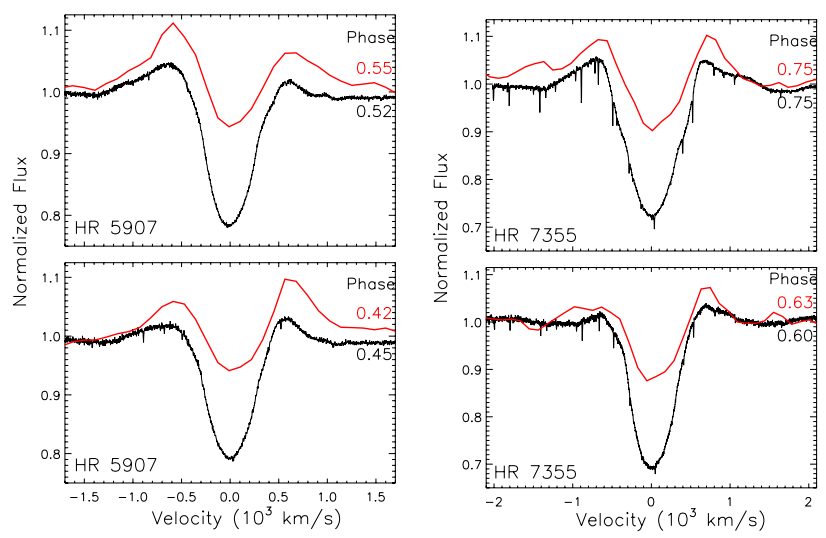

Fig. 2. Comparison of optical ( $\mathrm{H} \alpha$, black) and IR (Br10, red) spectral features. Left: HR 5907 at two different rotational phases, $\sim 0.45$ and $\sim 0.55$ (phases noted to the right). These two phases occur just before and just after the $\mathrm{H} \alpha$ emission maximum (Grunhut et al. 2012). Right: HR 7355 at phases $\sim 0.60$ and $\sim 0.75$. A peak in $\mathrm{H} \alpha$ emission occurs at phase 0.78 (Rivinius et al. 2013).

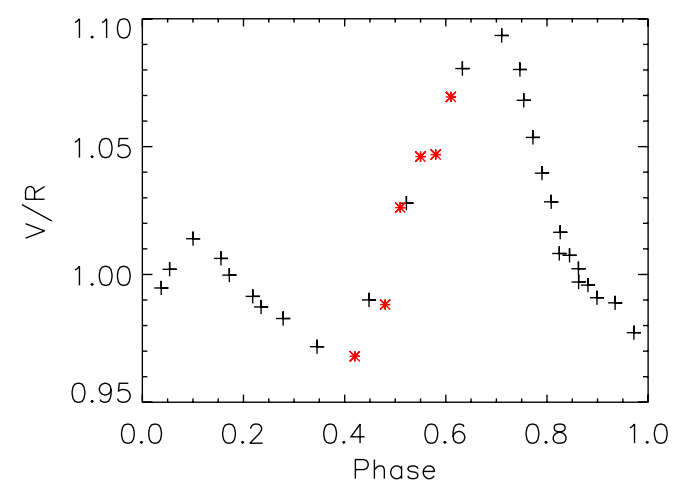

Fig. 3. V/R variations of observed emission in $\mathrm{H} \alpha$ (black pluses) and Br10 (red asterisks) spectral lines for HR 5907.

shows the $\operatorname{Br} 10, \operatorname{Br} 11, \operatorname{Br} 12$, and $\mathrm{Br} \gamma$ lines. While the data have a low resolution, the circumstellar emission is obvious at each rotational phase. The time series of HR 5907 (Fig. 1, top) only covers $20 \%$ of the rotation period, but the spectral lines show obvious variability in the strengths of the red and blue emission peaks, best viewed in the $\mathrm{Br} 10$ line. This variation is similar to behavior seen in $\mathrm{H} \alpha$ line profiles of both of our targets. Although the observations of HR 7355 (Fig. 1, bottom) only cover $10 \%$ of the rotational phase, the variability is easily visible.

The goal of this fundamental study is to determine whether spectral features in the IR are useful for detecting emission from a magnetosphere. One way to test this, given that we have precise information about the rotational modulation of the magnetic field of the star, is to compare the characteristics of the IR features with those of the optical features, namely $\mathrm{H} \alpha$ emission. As a result of telluric complications, we chose to focus our analysis on the strongest, cleanest feature in each of the spectra, the Br10 line at $1.737 \mu \mathrm{m}$. Figure 2 shows two examples of the Br10 (red) and $\mathrm{H} \alpha$ (black) lines at similar phases for HR 5907 and HR 7355. Comparing the heights of the red vs. blue peaks of the lines, corresponding optical and IR line profiles mimic each other in relative heights. For HR 5907, phase $\sim 0.45$ displays a stronger red peak, while phase $\sim 0.55$ shows a stronger blue peak. This is consistent with the maximum $\mathrm{H} \alpha$ emission occurring at phase $\sim 0.5$, as shown in Fig. 3 of Grunhut et al. (2012). The profile for HR 7355 at 0.75 shows two nearly equal peaks, which is
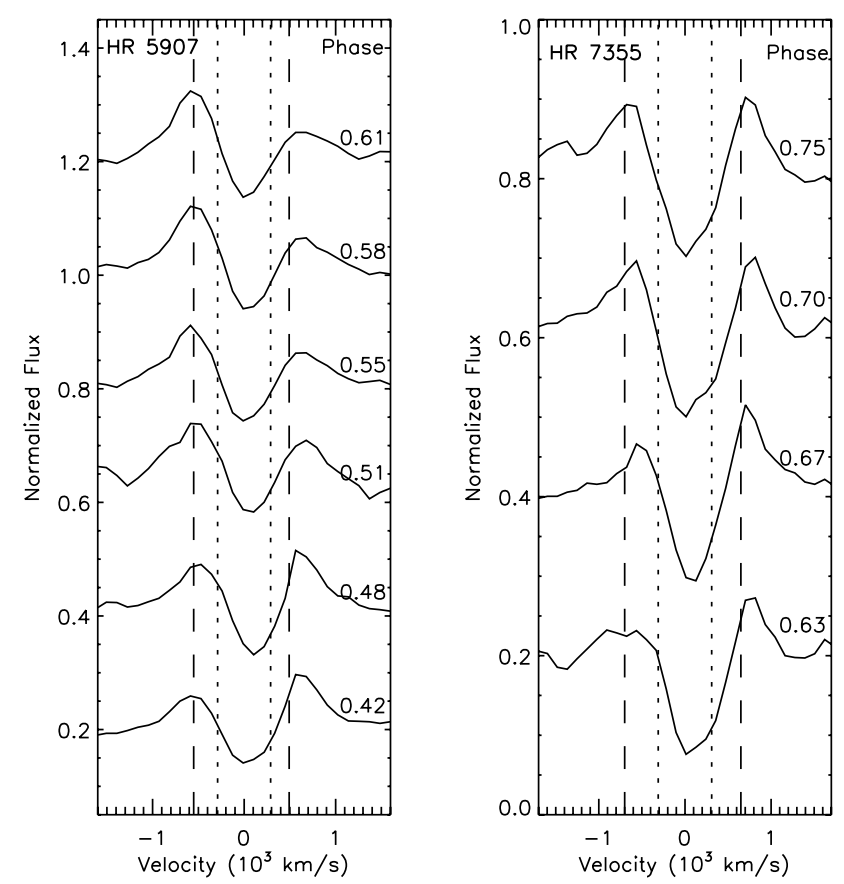

Fig. 4. Time-series of the Br10 line of HR 5907 (left) and HR 7355 (right). Stellar rotational phases for each spectrum are indicated to the right. The dotted lines indicate stellar rotation velocities derived from optical spectroscopy, while dashed lines indicate the peak emission found for $\mathrm{H} \alpha$ (Grunhut et al. 2012; Rivinius et al. 2013).

expected given that its $\mathrm{H} \alpha$ peak occurs at phase 0.78 (Rivinius et al. 2013). We also note that Fig. 1 suggests an effect whereby the peaks of the emission in $\operatorname{Br} \gamma$ trail behind the pattern observed in Br10. However, this is probably a result of the telluric contamination in $\operatorname{Br} \gamma$. To further reiterate the similarity of the emission peak behavior in the two wavelength domains, Fig. 3 shows $\mathrm{V} / \mathrm{R}$ variations for $\mathrm{HR} 5907$, measured from both $\mathrm{H} \alpha$ and $\mathrm{Br} 10$ (red asterisks) lines. The agreement between the two diagnostics is striking. An analogous plot for HR 7355 was not possible given that we have fewer IR data points, but also because the emission in this star is weaker than for HR 5907, and thus more difficult to precisely measure outside of maximum phases.

Figure 4 demonstrates that the bulk of the emitting plasma, in both cases, is located far beyond the stellar radius (dotted line) and is comparable with the value found for the peak in the optical from $\mathrm{H} \alpha$ (dashed lines, Grunhut et al. 2012; Rivinius et al. 2013). Although there may appear to be a slight difference in the location of the IR material (the red peaks appear systematically at higher velocities), this may just be a consequence of the low resolution, and higher-quality data are necessary to confirm or contradict this. As the circumstellar material responsible for the observed emission is in forced co-rotation, the location of the material is directly related to the projected velocity. For both stars, the bulk of the material is thus found at $\sim 2 R_{\star}$, coincident with the location of the Kepler corotation radius. This result agrees with MHD simulations (see, e.g., ud-Doula et al. 2008, Fig. 9).

Based on this evidence, we assert the equivalency of the hydrogen recombination lines in the IR with those in the optical for diagnosing the presence of a magnetic field.

Recently, spectropolarimetric observations of one of the IR emission magnetic candidates of Eikenberry et al. (2014) indeed revealed a strong magnetic field (Sikora et al. 2015), quite similar to that of $\sigma$ Ori E. This result provides confirmation 
that massive stars with magnetic fields can be detected through IR emission features, although no IR variability study has been performed in this case. Additional comparison of the IR and optical profiles demonstrates that there is far less contribution from absorption profiles in the IR, revealing more clearly emission from the magnetosphere. This attribute is significant, considering that Najarro et al. (2011) have shown that the hydrogen lines in the IR are more sensitive to low mass-loss rates, detecting rates ten times lower than measurable by $\mathrm{H} \alpha$ emission, and is particularly important if the physical parameters suggest a detectable magnetosphere (given the guidelines determined by ud-Doula \& Owocki 2002; Petit et al. 2013), but optical diagnostics fail. For these situations, IR observations may be more effective at detecting and characterizing the properties of circumstellar material, especially for low-density environments.

Given the positive detection of IR emission features in known magnetic stars, we have already begun subsequent work to obtain higher resolution data to study the structure of known magnetospheres with the aim to precisely determine the physical parameters of the emitting plasma, using modern techniques such as tomography (Grunhut et al. 2013). We have also obtained data to study the effects mentioned in the previous paragraph, whereby lower density environments, while undetected in optical features, may be observed in IR features. This study would then allow characterizing a whole new set of magnetospheres with varied physical parameters, which in turn would motivate further development of magnetic models and theory. We also aim to understand the IR line profiles of known magnetic O-stars and to determine what information may be obtained from such features so that we can better understand the interaction between the strong stellar wind and the magnetic field. Fundamentally, the research presented here serves as the initial step towards modernizing the study of magnetic massive stars, with these few example studies constituting the beginning of a larger shift in perspective.

As many hot stars are hidden in star-forming regions and other areas that are highly obscured from optical and UV observations, such as the Galactic center (see, e.g., Wachter et al. 2010; Gvaramadze et al. 2010), IR spectroscopy presents a solution for both identifying and studying new magnetic hot stars and their circumstellar environments. In fact, studying magnetic stars in the IR may (with higher resolution and $\mathrm{S} / \mathrm{N}$ ) be able to determine the consequences of the environment on the incidence of magnetism, that is, various types stellar clusters, the Galactic center vs. within the Galactic disk, high vs. low metallicity, etc.

In the future, this technique can be used to identify targets for IR spectropolarimetry, both Galactic and extragalactic, to identify and determine physical characteristics of the magnetosphere, and hence infer some properties of the magnetic field itself. These new technical capabilities will also be crucial in searching for young O-type stars that only become visible once their natal material is shed, providing unprecedented clues to the origin of magnetism in the most massive stars. Clearly, these types of observations are imperative to reveal and characterize magnetic massive stars, particularly undiscovered objects in heavily shrouded areas.

\section{Conclusions}

While the study presented here contains results from only two stars extracted from low-resolution data, the concepts and findings are applicable to the entire field of magnetic hot stars. In particular, we stress that IR spectra, particularly the $H$-band brackett series, are ideal for studying hot-star magnetospheres, given the lower contribution of stellar flux at these wavelengths.
We can detect this material and its variability even with lowresolution spectra. The identified IR spectral features match those found in optical spectra well, including the derived location of the detected material. We are currently extending this work to higher resolution spectra with the aim to study known magnetic stars, with the intent to apply this knowledge to candidate stars in regions inaccessible to UV and optical observations.

IR spectroscopy may be an essential tool for detecting and studying lower density environments around magnetic stars, for detecting magnetic candidates in the Galactic center and starforming regions, for studying young $\mathrm{OB}$ stars, and for increasing the number of known magnetic stars to determine basic formation properties. There is an urgency to understand and develop tools and techniques in the IR not only because massive star magnetism is a rapidly growing field, but also because of the innovative development of IR spectropolarimeters, such as SPIRou (Artigau et al. 2014) and CRIRES+ (Dorn et al. 2014), which will require in-depth knowledge of the features and behavior of magnetic diagnostics in the IR and strategies for best exploiting the results to determine physical properties. Ultimately, observations and models of these magnetospheres in multiple wavebands are crucial for understanding hot stars, their winds, and the effect of magnetic fields on the star and its surroundings.

Acknowledgements. We thank J. Sundqvist for useful discussions. We also thank Th. Rivinius for supplying UVES spectra of HR 7355. M.K. acknowledges financial support from GA ČR (grant 14-21373S) and from the European Structural Funds grant for the Centre of Excellence "Dark Matter in (Astro)particle Physics and Cosmology". The Astronomical Institute Ondřejov is supported by the project RVO:67985815. OSIRIS is a collaborative project between the Ohio State University and Cerro Tololo Inter-American Observatory (CTIO) and was developed through NSF grants AST 90-16112 and AST 92-18449.

\section{References}

Artigau, É., Kouach, D., Donati, J.-F., et al. 2014, in SPIE Conf. Ser., 9147, 15 Babel, J., \& Montmerle, T. 1997, A\&A, 323, 121

Borra, E. F., \& Landstreet, J. D. 1979, ApJ, 228, 809

Donati, J.-F., Babel, J., Harries, T. J., et al. 2002, MNRAS, 333, 55

Dorn, R. J., Anglada-Escude, G., Baade, D., et al. 2014, The Messenger, 156, 7

Eikenberry, S. S., Chojnowski, S. D., Wisniewski, J., et al. 2014, ApJ, 784, L30

Eisenstein, D. J., Weinberg, D. H., Agol, E., et al. 2011, AJ, 142, 72

Gagné, M., Oksala, M. E., Cohen, D. H., et al. 2005, ApJ, 628, 986

Granada, A., Arias, M. L., \& Cidale, L. S. 2010, AJ, 139, 1983

Grunhut, J. H., Rivinius, T., Wade, G. A., et al. 2012, MNRAS, 419, 1610

Grunhut, J., Townsend, R., \& Wade, G. 2013, in Massive Stars: From alpha to Omega, 69

Gvaramadze, V. V., Kniazev, A. Y., \& Fabrika, S. 2010, MNRAS, 405, 1047

Hanson, M. M., Kudritzki, R.-P., Kenworthy, M. A., Puls, J., \& Tokunaga, A. T. 2005, ApJS, 161, 154

Henrichs, H. F., et al. 1993, in New Perspectives on Stellar Pulsation and Pulsating Variable Stars, eds. J. M. Nemec, \& J. M. Matthews, IAU Colloq., 139,186

Landstreet, J. D., \& Borra, E. F. 1978, ApJ, 224, L5

Lenorzer, A., de Koter, A., \& Waters, L. B. F. M. 2002, A\&A, 386, L5

Najarro, F., Hanson, M. M., \& Puls, J. 2011, A\&A, 535, A32

Oksala, M. E., Wade, G. A., Marcolino, W. L. F., et al. 2010, MNRAS, 405, L51

Oksala, M. E., Wade, G. A., Townsend, R. H. D., et al. 2012, MNRAS, 419, 959

Petit, V., Owocki, S. P., Wade, G. A., et al. 2013, MNRAS, 429, 398

Rivinius, T., Štefl, S., Townsend, R. H. D., \& Baade, D. 2008, A\&A, 482, 255

Rivinius, T., Szeifert, T., Barrera, L., et al. 2010, MNRAS, 405, L46

Rivinius, T., Townsend, R. H. D., Kochukhov, O., et al. 2013, MNRAS, 429, 177

Ruschel-Dutra, D., Riffel, R., Ducati, J. R., \& Pastoriza, M. 2011, PASP, 123, 1004

Sikora, J., Wade, G. A., Bohlender, D. A., et al. 2015, MNRAS, submitted

Silvester, J., Kochukhov, O., \& Wade, G. A. 2014, MNRAS, 440, 182

Trigilio, C., Leto, P., Umana, G., Leone, F., \& Buemi, C. S. 2004, A\&A, 418, 593

ud-Doula, A., \& Owocki, S. P. 2002, ApJ, 576, 413

ud-Doula, A., Owocki, S. P., \& Townsend, R. H. D. 2008, MNRAS, 385, 97

Wachter, S., Mauerhan, J. C., Van Dyk, S. D., et al. 2010, AJ, 139, 2330 